Annales Geophysicae (2002) 20: 1699-1710 (C) European Geosciences Union 2002

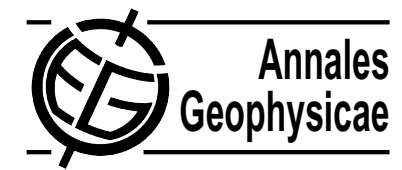

\title{
Cluster magnetic field observations at a quasi-parallel bow shock
}

\author{
E. A. Lucek ${ }^{1}$, T. S. Horbury ${ }^{1}$, M. W. Dunlop ${ }^{1}$, P. J. Cargill ${ }^{1}$, S. J. Schwartz ${ }^{2}$, A. Balogh ${ }^{1}$, P. Brown ${ }^{1}$, C. Carr ${ }^{1}$, \\ K.-H. Fornacon ${ }^{3}$, and E. Georgescu ${ }^{4,5}$ \\ ${ }^{1}$ Space and Atmospheric Physics, The Blackett Laboratory, Imperial College, London, UK \\ ${ }^{2}$ Astronomy Unit, Queen Mary, University of London, London, UK \\ ${ }^{3}$ Institut für Geophysik und Meteorlogie, Technische Universität Braunschweig, Germany \\ ${ }^{4}$ Max-Planck-Institut für Extraterrestrische Physik, Garching, Germany \\ ${ }^{5}$ Institut of Space Sciences, Bucharest, Romania
}

Received: 14 September 2001 - Revised: 24 June 2002 - Accepted: 17 July 2002

\begin{abstract}
We present four-point Cluster magnetic field data from a quasi-parallel shock crossing which allows us to probe the three-dimensional structure of this type of shock for the first time. We find that steepened ULF waves typically have a scale larger than the spacecraft separation $(\sim 400-1000 \mathrm{~km})$, while SLAMS-like magnetic field enhancements have different signatures in $|\mathbf{B}|$ at the four spacecraft, suggesting that they have a smaller scale size. In the latter case, however, the angular variations of $B$ are similar, consistent with the spacecraft making different trajectories through the same structure. The field enhancements have different orientations relative to a model bow shock normal, which might arise from different degrees of deceleration and deflection of the surrounding solar wind plasma. The observed rotation of the magnetic field rising from a direction approximately parallel to the model bow shock normal to a direction more perpendicular to the model normal across the field enhancement is consistent with previously published results. Successive magnetic field enhancements or ULF waves, and the leading and trailing edges of the same structure, are found to have different orientations.
\end{abstract}

Key words. Interplanetary physics (planetary bow shocks)

\section{Introduction}

The characteristics of collisionless planetary bow shocks are strongly dependent on the angle between the upstream magnetic field and the bow shock normal, $\theta_{B n}$. When $\theta_{B n}$ exceeds $45^{\circ}$, i.e. the shock normal is quasi-perpendicular to the upstream magnetic field, the shock tends to have a sharp transition. Magnetic field observations typically show a short ramp in $|\mathbf{B}|$, possibly with a "foot" ahead of the ramp, an overshoot after the ramp, or a high frequency wave train upstream of the ramp (e.g. Scudder et al., 1986). Quasiperpendicular shocks have been studied in detail over many years and recently Cluster data have been used to examine

Correspondence to: E. A. Lucek (e.lucek@ic.ac.uk) the structure of these shocks in three dimensions (Horbury et al., 2001, 2002).

When $\theta_{B n}$ is less than $\sim 45^{\circ}$, particles are able to escape upstream, generating and interacting with a variety of waves situated in a foreshock region (e.g. Le and Russell, 1992a, b), and the shock transition is found to be far more extended and unsteady (e.g. Greenstadt et al., 1982). A combination of satellite observations (e.g. Gosling et al., 1989; Thomsen et al., 1990; Schwartz et al., 1992) and simulation work (e.g. Burgess, 1989; Thomas et al., 1990; Scholer, 1993) have led to a picture of a shock undergoing cyclic reformation, composed of a patchwork of magnetic field enhancements called SLAMS (Short, Large-Amplitude, Magnetic Structures) and regions between the SLAMS where the magnetic field is disturbed and the plasma is partially thermalised (e.g. Schwartz, 1991; Schwartz and Burgess, 1991; Scholer and Burgess, 1992; Giacalone et al., 1994).

SLAMS are identified as magnetic field signatures when the magnetic field magnitude is enhanced over the undisturbed field by at least a factor of 2.5 , with durations of the order of $10 \mathrm{~s}$ or so in the spacecraft frame. They have a relatively smooth profile and are, therefore, sometimes described as having a "near-monolithic" form. It has been suggested that they grow out of ULF waves in the foreshock region (Schwartz, 1991; Giacalone et al., 1993; Mann et al., 1994). They occur both in regions of ULF wave activity (isolated SLAMS) and within regions of stronger magnetic field pulsations, associated with decelerated and heated plasma (embedded SLAMS). They are found to propagate sunward in the plasma frame, but are convected anti-sunward by the solar wind. They display mixed polarisation, biased towards right-hand polarised signatures (in the spacecraft frame), often with the remnant of a left-handed (in the spacecraft frame), high frequency whistler wave on the leading edge, similar to that found at the lower amplitude, steepened ULF waves observed in the foreshock (Schwartz et al., 1992) commonly called "shocklets".

Statistical studies of SLAMS also show that the magnetic field rotates from a direction approximately parallel to a 
model bow shock normal (upstream), to a direction closer to perpendicular, to a model bow shock normal (downstream), across the magnetic field enhancement (e.g. Mann et al., 1994). This magnetic field rotation changes the particle behaviour at the SLAMS, since locally the magnetic field is similar to a quasi-perpendicular shock, which can lead to gyrating ions downstream of the SLAMS, which scatter and partially thermalise the plasma. The study by Mann et al. (1994) of 18 isolated SLAMS also showed that the minimum variance direction, interpreted as the propagation direction, lay within $14.6 \pm 8.7^{\circ}$ of a model bow shock normal.

Observational studies of the quasi-parallel bow shock have been complemented by simulations which have explored the behaviour of particles at structures within the shock transition, and have shown that structures similar to SLAMS are generated (e.g. Scholer, 1993; Giacalone et al., 1994). A simulation of the foreshock region by Dubouloz and Scholer (1995) showed that SLAMS-like structures were generated when ULF waves, propagating against the solar wind flow, interacted with a high density, low energy diffuse ion population, as suggested by Giacalone et al. (1993) from a study of AMPTE data. Simulations have also shown that these structures typically evolve very quickly, on subsecond time scales. Giacalone et al. (1994) compared the results from a one-dimensional hybrid code to simulated multi-spacecraft observations through the same shock transition, and found that in this case spacecraft separated by only $188 \mathrm{~km}$ along the nominal shock normal direction saw significantly different signatures. Scale sizes of SLAMS-like structures occurring in simulations (e.g. Dubouloz and Scholer, 1995) have been found to be of the order of 15 ion inertial lengths perpendicular to the shock normal, and a few tens of ion inertial lengths parallel to the shock normal. The simulation work described by Dubouloz and Scholer (1995) predicts that although SLAMS grow from the upstream ULF waves, which observationally have scale sizes of $\sim 1 R_{E}$ (Le and Russell, 1990), SLAMS should have smaller scale sizes as differential slowing of the incident flow was found to lead to fragmentation of the ULF waves.

The structures within the quasi-parallel shock transition, therefore, are expected to be complex, possibly with different scale lengths in different directions (Schwartz and Burgess, 1991; Dubouloz and Scholer, 1995), but single or dual spacecraft observations are not sufficient to probe their three-dimensional form. For the first time four-point observations are available from the Cluster mission, launched in 2000 into a polar orbit, with an apogee of $19.6 R_{E}$ and perigee of $4 R_{E}$. The choice of orbit means that bow shock crossings are biased to high latitude locations when apogee is near noon, although low latitude crossings do occur when apogee lies in the flanks of the magnetosphere.

The bow shock is one of the key regions for study using Cluster data. Simultaneous observations by spacecraft pairs with different separation vectors relative to the plasma flow, expected bow shock normal, and magnetic field allow, in principal, the three-dimensional characteristics of structures within the shock transition to be studied. We use the observations with caution, however, since simulations (e.g. Giacalone et al., 1994) suggest that features within the shock evolve rapidly, on time scales of a few seconds or less, and might not be planar on scales of a few hundred to a thousand kilometres, which is approximately the scale of the Cluster tetrahedron during the observations made to date.

This study describes some of the characteristics of the magnetic field structures during the shock transition, and draws entirely on data from the four FGM instruments (Balogh et al., 2001). Although we concentrate on SLAMS, we also discuss the observational characteristics of upstream shocklets, which have a steepened leading edge (relative to their plasma frame propagation direction) and often also an associated whistler wave, for comparison with the SLAMS. A small number of intervals are discussed in detail, where we are able to estimate a propagation direction using both a method based on the relative timing of a feature at the four spacecraft, and minimum variance analysis. We also discuss minimum variance analysis of the upstream ULF wave field. SLAMS-like structures are distinguished from the magnetic signatures surrounding hot flow anomalies (HFA) (Schwartz, $1985,1991)$ by their plasma signatures, and so here we cannot definitely identify SLAMS. We do, however, find magnetic field structures with characteristics that are consistent with previous observations of SLAMS.

\section{Analysis}

\subsection{Overview}

In this paper we concentrate on one shock transition, on 2 February 2001 at $\sim 21: 00$ UT, when Cluster was at a local time of $\sim 15: 00$ LT. The upstream solar wind observed at ACE, approximately one hour earlier, had a velocity of $\sim 405 \mathrm{~km} / \mathrm{s}$, rising to $\sim 430 \mathrm{~km} / \mathrm{s}$ at around 20:30 UT, implying a jump in the solar wind velocity at the Earth at about 21:30 UT. The magnetic field magnitude at ACE was similar to that observed at times when Cluster was sampling undisturbed solar wind plasma, $\sim 2.5 \mathrm{nT}$, and the number density at ACE was $\sim 2.8 \mathrm{~cm}^{-3}$. This implies an Alfvén Mach number for the bow shock of $\sim 12-13$ and a value for the ion inertial length in the undisturbed solar wind of $\sim 135 \mathrm{~km}$. Figure 1 shows an overview of spin averaged magnetic field data (at $4 \mathrm{~s}$ resolution) recorded by Cluster 4 . The shock transition, characterised by strongly compressive magnetic field variations, lasts for several hours, and there are a number of bursts of smaller amplitude upstream wave activity, characteristic of the foreshock (e.g. just before 23:00 UT).

We order the magnetic field observations through the shock transition by comparing the instantaneous magnetic field direction with a bow shock normal derived from the Peredo et al. (1995) model under average solar wind conditions (see Horbury et al., 2002). In a study of 48 quasiperpendicular bow shock crossings, Horbury et al. (2002) showed that there was good agreement, typically within $\sim 10^{\circ}$, between the model normal and the normal derived 


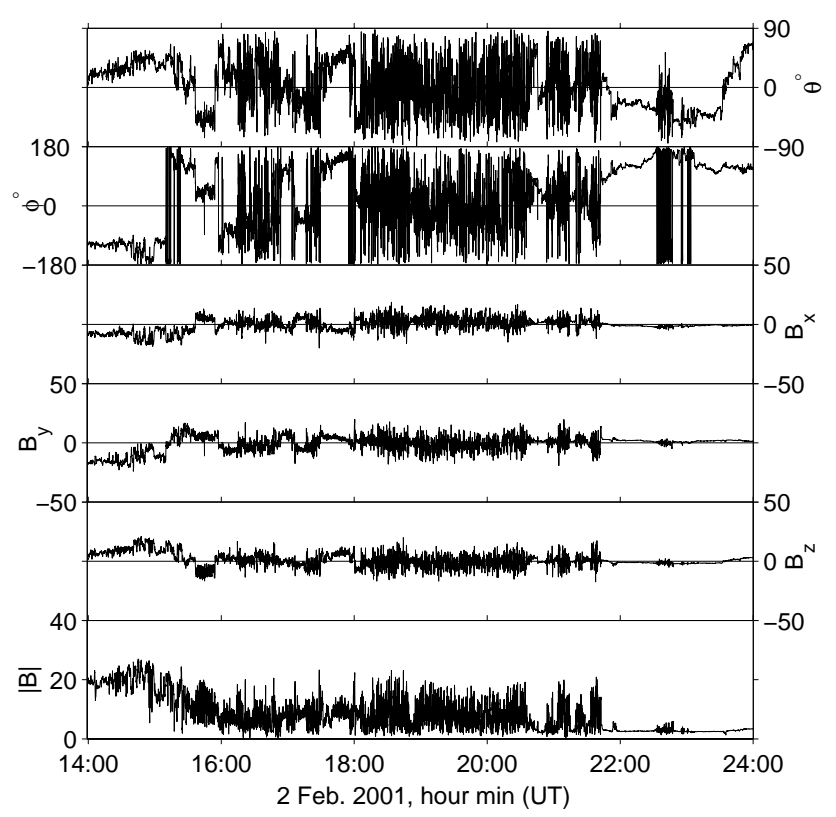

Fig. 1. Magnetic field data recorded by Cluster 4 through the quasiparallel shock on 2 February 2001. Data are displayed in the GSE coordinate system. Panels show magnetic field elevation $(\theta)$ and longitude $(\phi)$ angles in degrees, three magnetic field components $\left(B_{x}, B_{y}, B_{z}\right)$ and the magnetic field magnitude $|\mathbf{B}|$ in nT.

from the timing of the shock crossings at the four spacecraft. Using the simplifications outlined by Horbury et al. (2002), the model shock normal is likely to be accurate to within $10^{\circ}$, which is sufficient to demonstrate the trends in the magnetic field direction across the shock on 2 February 2001. Figure 2 shows the magnetic field data from Cluster 1 (in black) and ACE (in light blue) during the shock transition, together with the angle between the local magnetic field direction and the model bow shock normal (Pred $\theta_{B n}$ ) estimated using the simplified Peredo model. Detailed comparison of the Cluster and ACE measurements shows that throughout the interval of interest, the delay time between ACE and Cluster is not constant, and that there are several intervals where the correspondence is poor. In Fig. 2 the time delay is altered such that the data sets show a good correspondence at three clear discontinuities (at $\sim 20: 45, \sim 21: 40$ and $\sim 21: 50$ UT). When the two data sets are dissimilar, such as between 20:50 and 21:10 UT, ACE data are not plotted.

Figure 2 shows that in the quiet magnetic field region upstream of the shock transition (e.g. 21:45-22:30 UT), $\theta_{B n}$ relative to the model shock normal is above $45^{\circ}$, indicating that this region will be likely to give rise to a quasi-perpendicular shock. During intervals of good correspondence within the shock transition, the magnetic field direction measured at ACE is typically consistent with a quasi-parallel shock configuration. The local field at Cluster has been modified by the shock process, and within the compressive pulsation regions the values of $\theta_{B n}$ are more scattered, and tend to be biased towards $90^{\circ}$. Such behaviour is expected from previous studies which showed that across SLAMS structures,

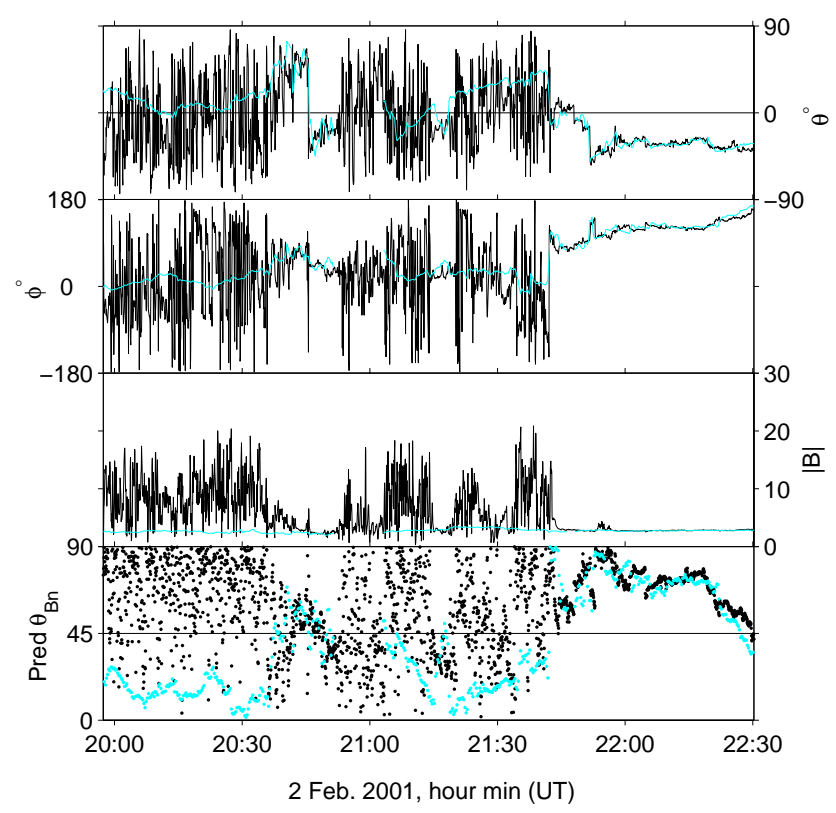

Fig. 2. The angular variations of the spin-averaged magnetic field at Cluster 1 (black) and upstream at ACE (light blue). Panels show magnetic field elevation $(\theta)$ and longitude $(\phi)$ angles in GSE coordinates, magnetic field magnitude $(|\mathbf{B}|)$ in $\mathrm{nT}$ and the angle between the model bow shock normal and the magnetic field (Pred $\left.\theta_{B} n\right)$. ACE data are lagged to give good correspondence between the magnetic field angles seen at ACE and at Cluster. When the data sets do not have clear features in common, ACE data are not plotted, for example, between $\sim 20: 50$ and 21:10 UT.

the magnetic field tended to rotate from an orientation nearly parallel to the model bow shock normal, to a direction more perpendicular to the shock normal (e.g. Schwartz et al., 1992; Mann et al., 1994).

Figure 3 shows the Cluster orbit and spacecraft tetrahedron configuration on 2 February 2001. A cut though a nominal model bow shock is shown on each panel. At the end of the day, when the shock is encountered, Cluster 2 is separated from the other three spacecraft by about $1000 \mathrm{~km}$ in the $X-$ $Y_{\mathrm{GSE}}$ plane, mainly in the $Y_{\mathrm{GSE}}$ direction, lying closer to the nose of the magnetosphere. The other three spacecraft are quite closely aligned with the orbit in the $X-Y_{\mathrm{GSE}}$ plane, although separated in $Z_{\mathrm{GSE}}$, with spacecraft separations of the order of 400-800 km. Cluster 1 lies at the highest $Z_{\mathrm{GSE}}$, and Cluster 2 and 3 lie further upstream than Cluster 4.

Figure 4 shows a section of the shock transition in more detail. This interval contains the clearest examples of SLAMS-like magnetic field enhancements, but unfortunately, there is poor correspondence between the ACE magnetic field and the Cluster data during this time: two discontinuities are observed at ACE (not shown), which would be expected to be seen in the Cluster data at approximately 20:50 UT and 21:03 UT, but neither are found to occur at Cluster.

Data from the four spacecraft are plotted, using the same colour scheme as for the orbit plot. It can be seen from 

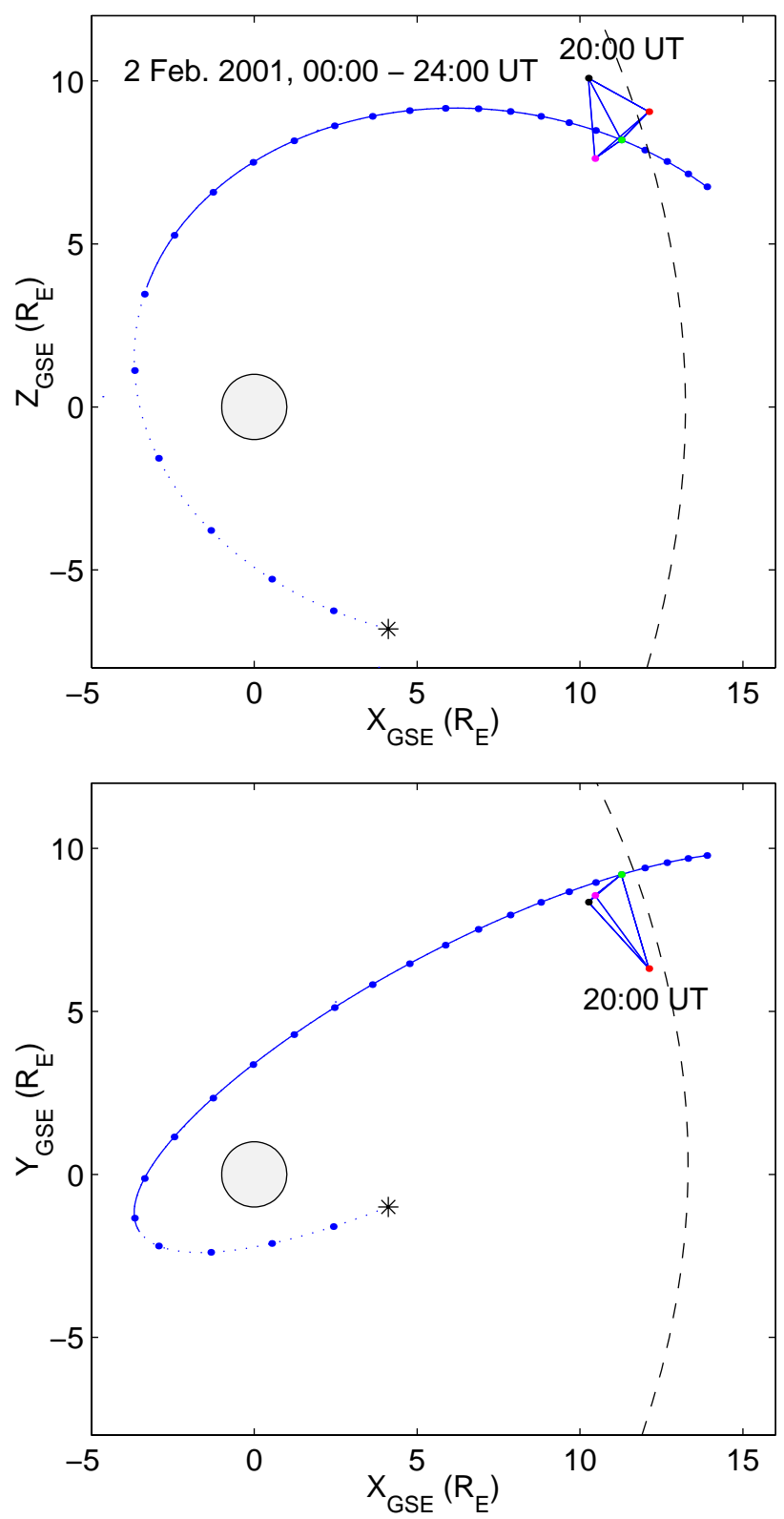

Fig. 3. The Cluster orbit track and spacecraft configuration projected onto the $X-Z_{\mathrm{GSE}}$ plane (top) and the $X-Y_{\mathrm{GSE}}$ plane (bottom). The orbit is shown in a dotted line when the third component is negative. The asterisk indicates the start of the interval. Filled circles indicate hourly markers along the orbit. The tetrahedron configuration is plotted on the orbit at around the time that the shock was encountered, expanded by a factor of 20 with the spacecraft indicated by black (Cluster 1), red (2), green (3) and magneta (4). A cut through a nominal bow shock location is shown on each panel for context.

this figure that the four spacecraft see similar features on large scales, but that there are significant differences at small scales, especially during the region of strong compressional activity after 21:03 UT. There is ultra-low frequency (ULF) wave activity from just after 20:55 UT, until 21:03 UT, which is interrupted by two large magnetic field magnitude en-

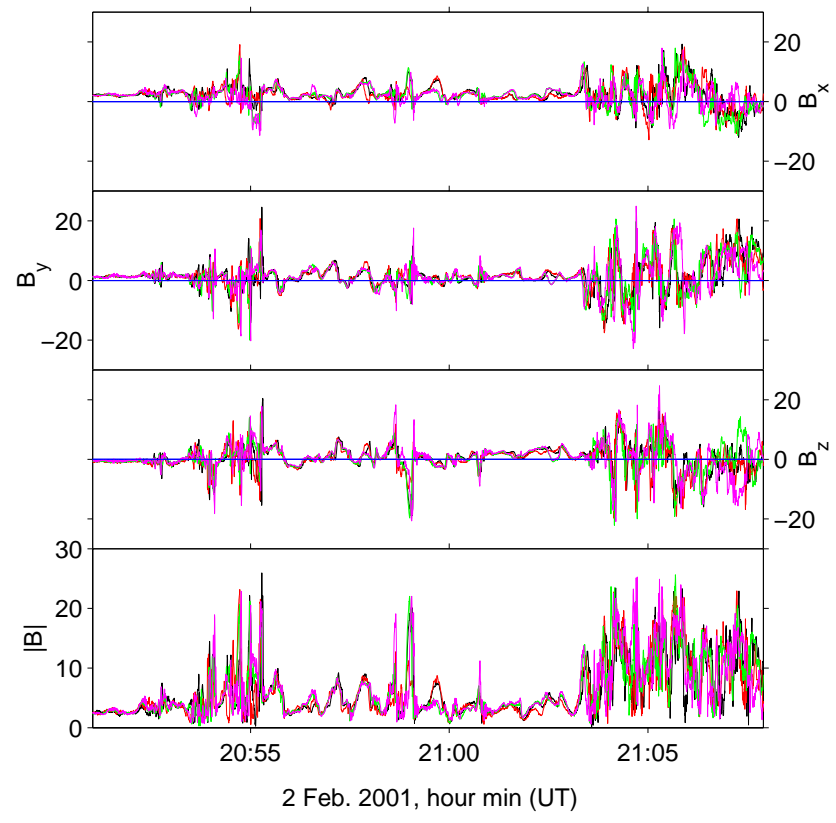

Fig. 4. Magnetic field data from the four spacecraft at 5 vectors/s from a region of the shock transition. The spacecraft are indicated by black (Cluster 1), red (2), green (3) and magenta (4). Panels show the three magnetic field components $\left(B_{x}, B_{y}, B_{z}\right)$ in GSE coordinates, and the magnetic field magnitude $(|\mathbf{B}|)$, all in nT.

hancements. Earlier, between 20:53 and 20:55 UT, there is another burst of activity which also has some magnetic field enhancements embedded in it. There are smaller differences between the magnetic fields measured at the four spacecraft during the ULF wave activity than during the magnetic pulsations, suggesting that the ULF waves are of larger scale, or evolving more slowly.

In the following sections we examine the correlation between the four Cluster spacecraft at a number of magnetic field structures within and upstream of the shock transition. We first analyse two magnetic field enhancements, one of which is embedded in the region of compressive magnetic field pulsations between 20:53 and 20:55 UT, and is, therefore, a candidate for an embedded SLAMS, and another which lies within the region of ULF wave activity, and is, therefore, a candidate for an isolated SLAMS. For comparison with the magnetic field enhancements, we examine examples of shocklets with associated upstream whistler waves. We also consider the characteristics of the ULF waves which occur in this interval, and lastly, we present a sharp transition into undisturbed solar wind.

\subsection{Magnetic field structures within the shock transition}

\subsubsection{Interval 1: 20:54:30-20:55:30 UT}

Figure 5 shows high resolution magnetic field data for two clear magnetic field magnitude enhancements which are embedded in further magnetic pulsations. The first is just before 20:54:45 UT, and the second is at 20:55:15 UT. Between 


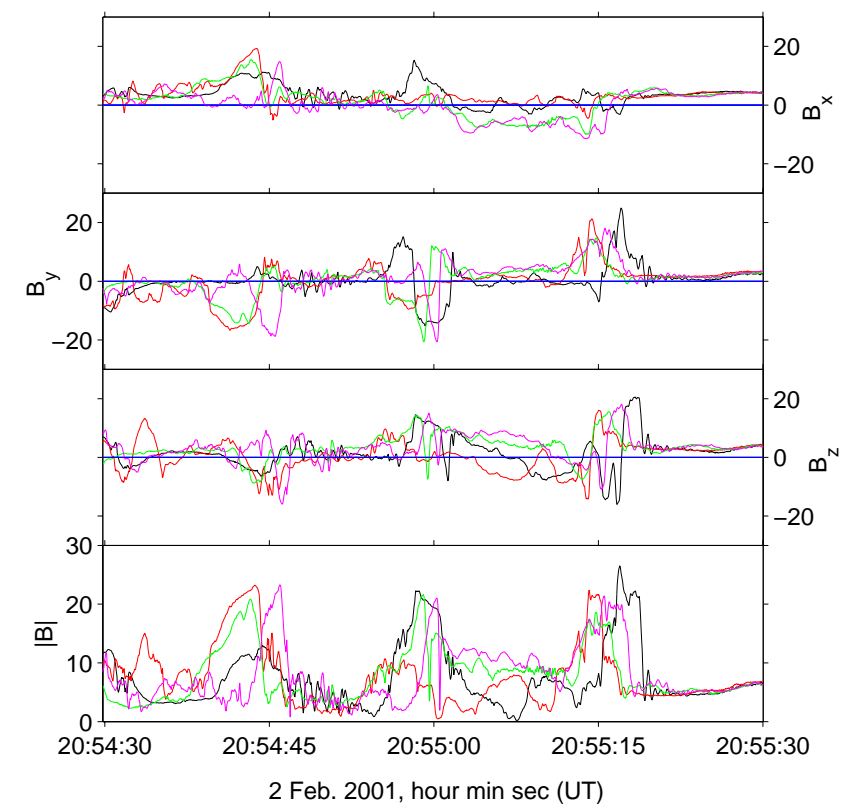

Fig. 5. Magnetic field data from the four spacecraft at 22 vectors/s showing magnetic field magnitude enhancements within a pulsation region. The format of the figure is as in Fig. 4.

them is a structure less well correlated between the spacecraft (at 20:55:00 UT). On these time scales, although the signatures at the four spacecraft are related, there are significant differences, suggesting that spatial changes occur on the order of the spacecraft separation, which at this time was between a few hundred and a thousand kilometres. Comparison of the ordering of the spacecraft shows that although the signatures appear to be convected towards the Earth (for example, the signature at Cluster 2 (red) or 3 (green) typically leads that at Cluster 4 (magenta)), the ordering of the spacecraft varies both between the leading and trailing edges of the structures, and between the two structures, even though they are only separated by $30 \mathrm{~s}$.

In the first case Cluster 1 (black) sees a much smaller enhancement, despite Cluster 2 and Cluster 4 observing signatures of a similar magnitude before and after the enhancement at Cluster 1. This suggests that the difference between Cluster 2 or 4 and Cluster 1 arises from spatial variations of the structure rather than temporal evolution. The orbit plot (Fig. 3) shows that Cluster 1 lies at a significantly larger $Z_{\mathrm{GSE}}$ than Cluster 2 or 4 . Comparison with the second clear example, at 20:55:15 UT, shows that in this case Cluster 1 sees a similar magnitude enhancement to the other three. Here, however, Cluster 2, 3 and 4 see a nearly simultaneous onset, while there is a delay before Cluster 1 sees the magnetic field rise. This implies that the surface of the structure first encountered by the spacecraft lies parallel to the plane containing Cluster 2, 3 and 4 . The spacecraft exit from the structure in a different order to the entry, suggesting that the orientations of the leading and trailing edges are different. It appears, therefore, that these structures are not planar on the

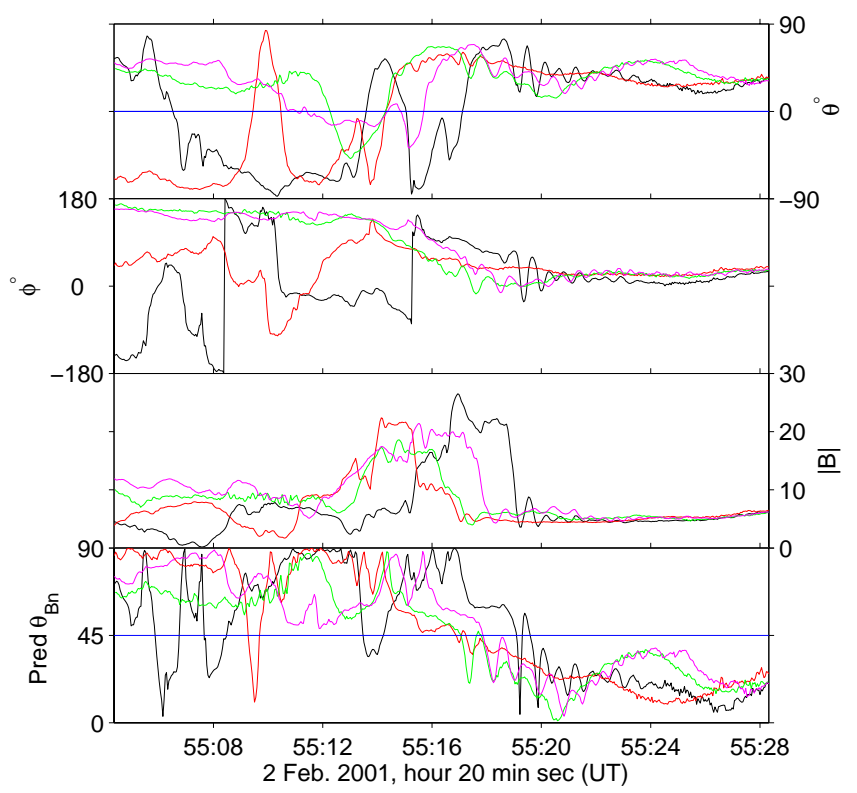

Fig. 6. The variation of the $\theta_{B n}$ angle predicted using the bow shock model normal across the magnetic field enhancement at 20:55:15 UT. Panels show the magnetic field elevation $(\theta)$ and longitude $(\phi)$ angles in GSE coordinates, the magnetic field magnitude $(|\mathbf{B}|)$ and $\theta_{B n}$.

scale of the tetrahedron. The timing differences between successive magnetic field enhancements might arise from different trajectories though the structures, or from the structures having different underlying orientations.

The second magnetic field enhancement, at 20:55:15 UT, is more similar between the four spacecraft. Figure 6 shows the change in the orientation of the magnetic field direction, relative to the model bow shock normal across this structure. The angle between the two directions is denoted $\theta_{B n}$. This figure shows that although there are differences between the magnetic field magnitude signatures at the four spacecraft, the behaviour of the magnetic field direction at the four spacecraft is quite similar. Moving from right (upstream of the enhancement) to left (through the enhancement): upstream of the leading edge, the magnetic field direction is within $45^{\circ}$ of the model bow shock normal; across the leading edge $\theta_{B n}$ rises, with a slight plateau at the end of the sharp ramp, then rises more quickly to a value near $90^{\circ}$ within the enhancement. The magnetic field magnitude at Cluster 2 has a feature similar to a shock foot, typically observed at quasiperpendicular shocks (e.g Scudder et al., 1986). Cluster 3 sees a similar feature, but it is less pronounced, and Cluster 4 and 1 observe short ramps, lasting for about $0.5 \mathrm{~s}$, on the leading edge. This variation could be explained by rapid temporal evolution of the leading edge. The smaller systematic variations in $\theta_{B n}$ just ahead of the ramp, which are clear in the data from Cluster 1 and 4, are caused by low amplitude high frequency waves which are left-handed in the spacecraft frame (with respect to the upstream magnetic field direction). This polarisation signature is consistent with right- 


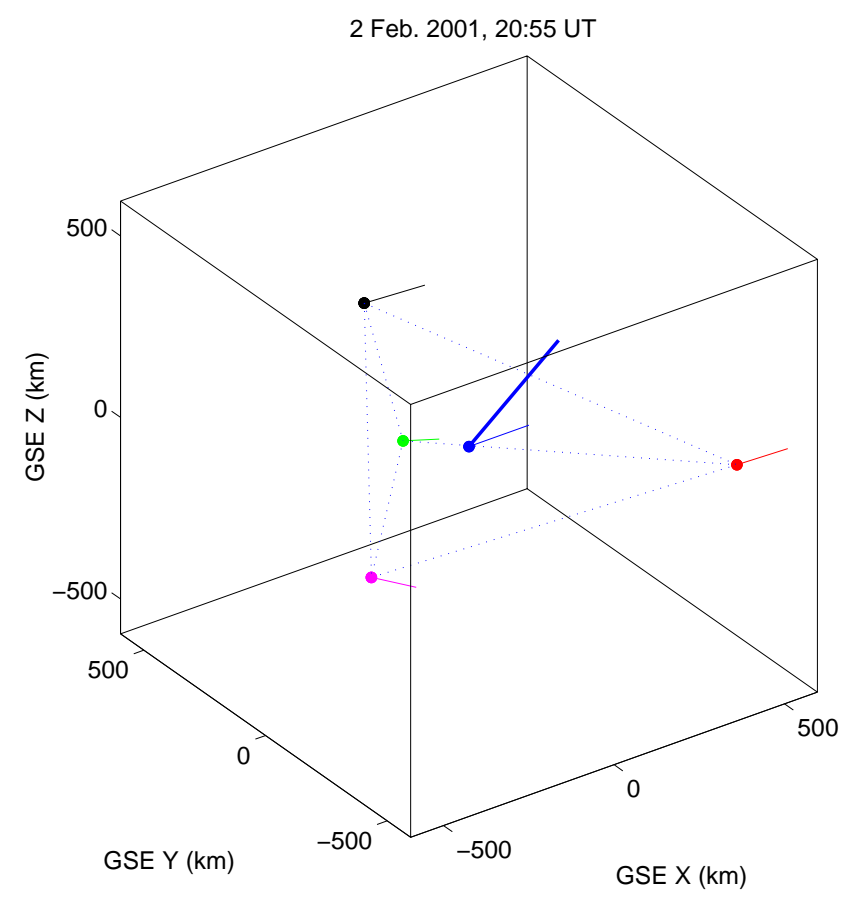

Fig. 7. A summary of the different estimates of the direction normal to the SLAMS surface: model normal (heavy blue line), timing derived normal (fine blue line), minimum variance directions estimated at the individual spacecraft (Cluster 1, 2, 3 and 4 represented by black, red, green and magenta, respectively)

handed whistler waves propatating sunward, but convected anti-sunward by the solar wind, hence reversing their polarisation signature, and this is a well-known feature of shocklets (e.g. Omidi and Winske, 1990; Le and Russell, 1992b) and SLAMS (e.g. Schwartz et al., 1992).

Minimum variance analysis (MVA) can be used to find the minimum variance direction, which for a plane wave can be interpreted as the propagation direction. We have compared the results of MVA for each of the four spacecraft for the magnetic field enhancement at 20:55 UT, and have also estimated a normal from the relative spacecraft timings of the ramp at the leading edge for comparison with MVA, using the method described by Schwartz (1998). When applying MVA to this event, we used intervals during which $|\mathbf{B}|$ was enhanced, excluding any intervals containing a whistler wave component, although it was found that the minimum variance directions were not strongly dependent on the data interval in this case. The normal from the discontinuity timing analysis is found to be $\boldsymbol{n}=(0.98,0.14,-0.10)$ in GSE coordinates, which is at an angle of $25^{\circ}$ to the model bow shock normal, $\boldsymbol{n}_{\boldsymbol{s} \boldsymbol{h}}=(0.90,0.34,0.27)$.

The minimum variance directions for the four spacecraft are found to be $\boldsymbol{e}_{1}=(0.91,0.13,-0.14) ; \boldsymbol{e}_{2}=(0.94,0.27$, $-0.22) ; \boldsymbol{e}_{3}=(0.81,0.39,-0.43) ; \boldsymbol{e}_{4}=(0.82,0.23,-0.52)$. The ratios between the intermediate and minimum eigenvalues for the MVA of data from each spacecraft exceed 4 in each case and are, therefore, well defined. The magnetic field enhancement is observed to be right-handed at all four space- craft. The angle between the minimum variance directions and the discontinuity timing normal is $2^{\circ}, 10^{\circ}, 26^{\circ}, 27^{\circ}$, for Cluster 1, 2, 3 and 4, respectively. Each minimum variance direction lies at greater angles to the model normal than the timing normal: $27^{\circ}, 28^{\circ}, 42^{\circ}$ and $47^{\circ}$, respectively.

The local magnetic field direction has been modified by the wave field, so it is useful to compare the propagation direction of the SLAMS structure, estimated either using timing analysis or MVA, with the undisturbed magnetic field direction. It is not possible in this case to use the magnetic field observed by ACE for this purpose, because there is poor correspondence between the ACE and Cluster observations during this interval, as described in the Analysis section. Therefore, the propagation direction was compared with the quiet magnetic field direction recorded just before the wave activity, although such a comparison is not ideal since the change in magnetic field character is likely to be associated with a change in underlying magnetic field direction. The propagation direction of the SLAMS structure was found to be at an angle of $50-75^{\circ}$ to the magnetic field direction in this region. The SLAMS structure, therefore, appears to be more closely aligned with the model bow shock normal than with the underlying magnetic field direction.

The different estimates for the normal to the SLAMS structure are summarised in Fig. 7, which shows the Cluster tetrahedron at the time when the magnetic field enhancement was observed. The positions of the four spacecraft are indicated by the coloured dots, and the centre of the tetrahedron is indicated by the blue point. The thick blue line indicates the direction of the model normal, and the fine blue line indicates the normal estimated from timing analysis. The minimum variance directions found for each individual spacecraft are indicated by the lines at the positions of the different spacecraft. The agreement between the minimum variance direction and the discontinuity timing analysis normal is closest for Cluster 1, which is furthest out of the $X-Y_{\mathrm{GSE}}$ plane. There is a systematic deflection of the minimum variance direction away from the timing normal between Cluster 2, 3 and 4, which supports the hypothesis that the structure is three-dimensional on the scale of the Cluster tetrahedron. Consequently, the timing derived normal is likely to give an average estimate of the normal over the tetrahedron scale. Comparison of the discontinuity timing normal with the bow shock model normal shows that they are separated by an angle of $\sim 25^{\circ}$, with the discontinuity timing vector being deflected southward. Although the minimum variance directions show quite a lot of scatter, all are deflected southward relative to the bow shock model normal. The angle separating the timing and model normals lies slightly outside the range found by Mann et al. (1994) of $\sim 15 \pm 9^{\circ}$, and several of the angles between the minimum variance directions and the model normal lie substantially outside this range. Mann et al. (1994), however, only considered isolated SLAMS, while this structure is consistent with an embedded SLAMS, which is associated with deflected and decelerated plasma flow relative to the undisturbed solar wind plasma, and such a deflection is consistent with the sense of discrepancy in the normal 


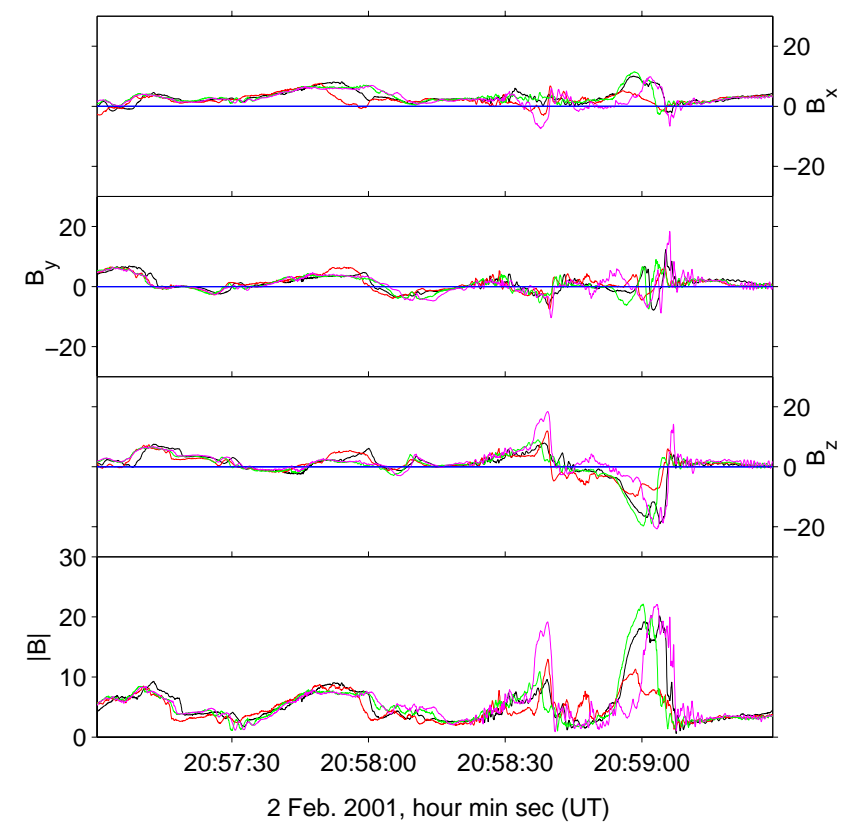

Fig. 8. Magnetic field data at 22 vectors/s in GSE coordinates, in the same format at Fig. 5.

directions.

\subsubsection{Interval 2: 20:57-21:00 UT}

The second interval of magnetic field enhancements to be considered in detail occurs four minutes after the interval discussed in the previous section. Figure 8 shows the high resolution magnetic field data in the same format as Fig. 5. The magnetic field enhancements stand out clearly from the surrounding ULF wave activity. In this interval the magnetic field enhancements are isolated from other strongly compressive magnetic field signatures, consistent with isolated SLAMS. The four spacecraft still see markedly different signatures. At the first structure (at 20:58:40 UT) Cluster 4, which lies earthward of Cluster 2 and 3, and further south than Cluster 1, observes a larger magnetic field magnitude enhancement, despite the signature occurring at approximately the same time in all four spacecraft, suggesting that the signature does not arise from temporal variations. Cluster 2 and 4 each observe a distinct leading edge, while there is a less clear leading edge seen by Cluster 1 and 3 .

At the second enhancement (at 20:59:00 UT), Cluster 4 observes a shorter enhancement than the other spacecraft, while Cluster 2 encounters a much smaller magnetic field magnitude than the other three. The variation in spacecraft ordering and observed signature is more likely to arise from spatial changes on scales of a few hundred to a thousand kilometres than temporal differences, since the structure neither systematically grows nor decays at successive spacecraft crossings. Figure 3 shows that Cluster 2 lies approximately $1000 \mathrm{~km}$ from the other three spacecraft, separated almost parallel to the nominal shock surface. In this case then, Clus-

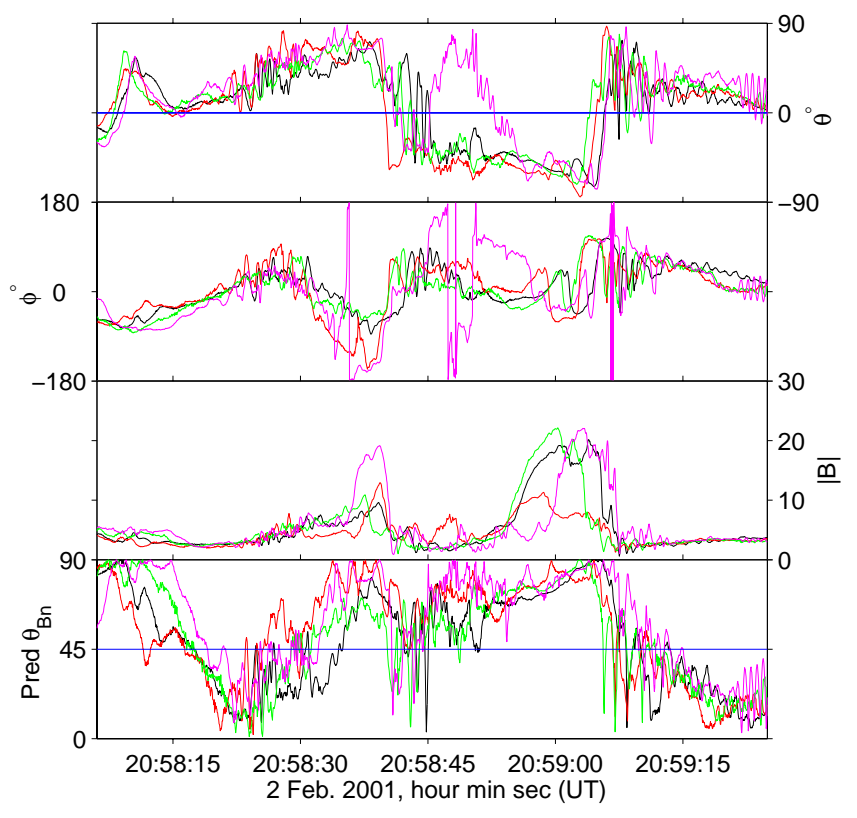

Fig. 9. The variation of the $\theta_{B n}$ angle predicted using the bow shock model normal across the two magnetic field enhancements during interval 2. Panels have the same format as in Fig. 6.

ter 2 might make a significantly different trajectory through the structure than the other three spacecraft, and the observed signature arises from spatial variations perpendicular to the nominal shock normal.

Figure 9 is in the same format as Fig. 6 and shows the magnetic field direction, magnetic field magnitude, and the angle of the local magnetic field relative to a model bow shock normal across the two magnetic field enhancements. Although Cluster 2 observes a smaller magnetic field magnitude at the second field enhancement (at $\sim 20: 59$ UT), the angular variation through both structures is very similar to that seen by the other three spacecraft, which is in good agreement, especially for the clearer of the two magnetic field enhancements. As in the previous example, the magnetic field upstream makes an angle to the model bow shock normal of less than $45^{\circ}$. Then, moving from right to left on Fig. 9, through the region with a high frequency wave (between $\sim 20: 59: 05$ and 20:59:20 UT) and across the leading edge, the magnetic field rotates until this angle becomes nearly $90^{\circ}$. The magnetic field retains this direction through the region where $|\mathbf{B}|$ is high. The angular variations in the region between the two magnetic field enhancements are much more irregular, suggesting that processes are occurring on smaller scales during that interval. During the smaller magnetic field enhancement, however, the local magnetic field once again rotates to make an angle of nearly $90^{\circ}$ to the model bow shock normal. The similarity of the angular signatures seen by Cluster 2 to those seen by the other spacecraft shows that the shape of the structure, measured by the magnetic field direction, is preserved over the separation distance to Cluster 2, despite the difference in magnetic field magnitude. 


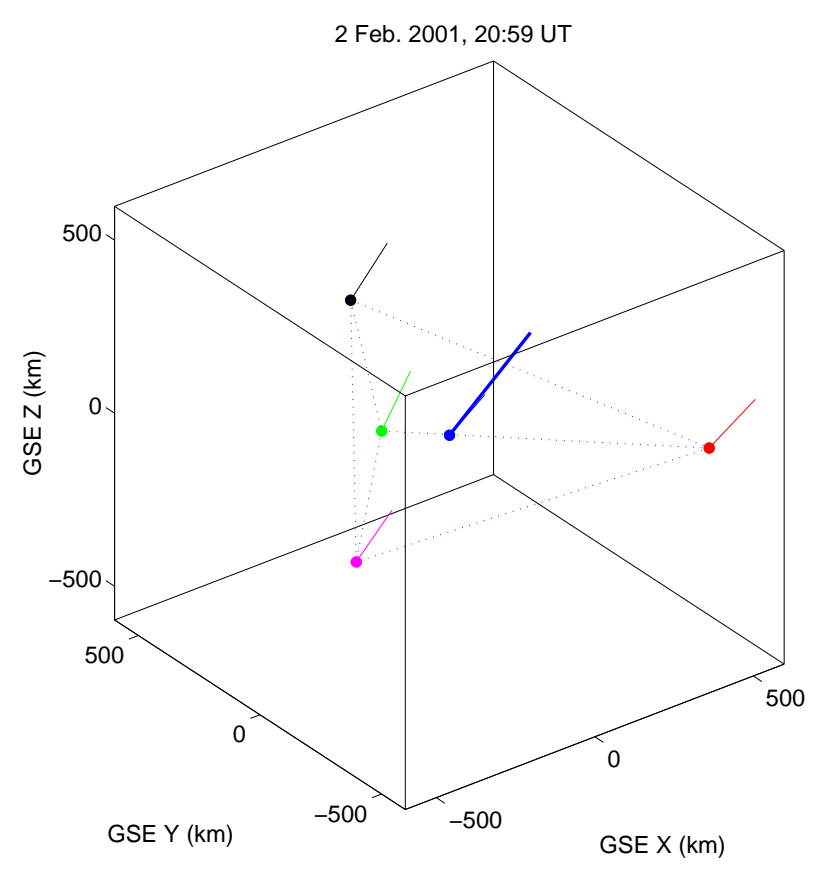

Fig. 10. Summary of the different estimates of the normal to the SLAMS at 20:59 UT, in the same format as Fig. 7.

Since the $|\mathbf{B}|$ signature at Cluster 2 is significantly different from that at Cluster 1, 3 and 4, discontinuity timing analysis cannot be applied to the $|\mathbf{B}|$ profile. Using the similarity of the angular variations, however, discontinuity timing analysis (not shown) was applied to the $\theta$ signature, giving a normal direction which agreed with the model normal within $13^{\circ}$ and with the minimum variance directions from the four spacecraft within $20^{\circ}$. The different estimates for the direction normal to the SLAMS are shown in Fig. 10, using the same format as in Fig. 7. In this case minimum variance analysis applied to the data from the four spacecraft gives minimum variance directions which agree with the model normal within $10^{\circ}$. This is consistent with the results found for isolated SLAMS by Mann et al. (1994). The better agreement between the calculated normal and minimum variance directions and the model normal might arise from the isolated nature of the enhancements which, if isolated SLAMS, are less likely to be associated with significant deceleration and deflection of the solar wind flow. A better agreement, therefore, would be expected between the orientation of the structure and the model bow shock normal.

Once again the different estimates of the SLAMS propagation direction were compared with the magnetic field direction just before this interval, and again the angular separation of these vectors was found to be large: in the range $60-75^{\circ}$, suggesting that the SLAMS was more closely aligned with the model bow shock normal than the background magnetic field direction.

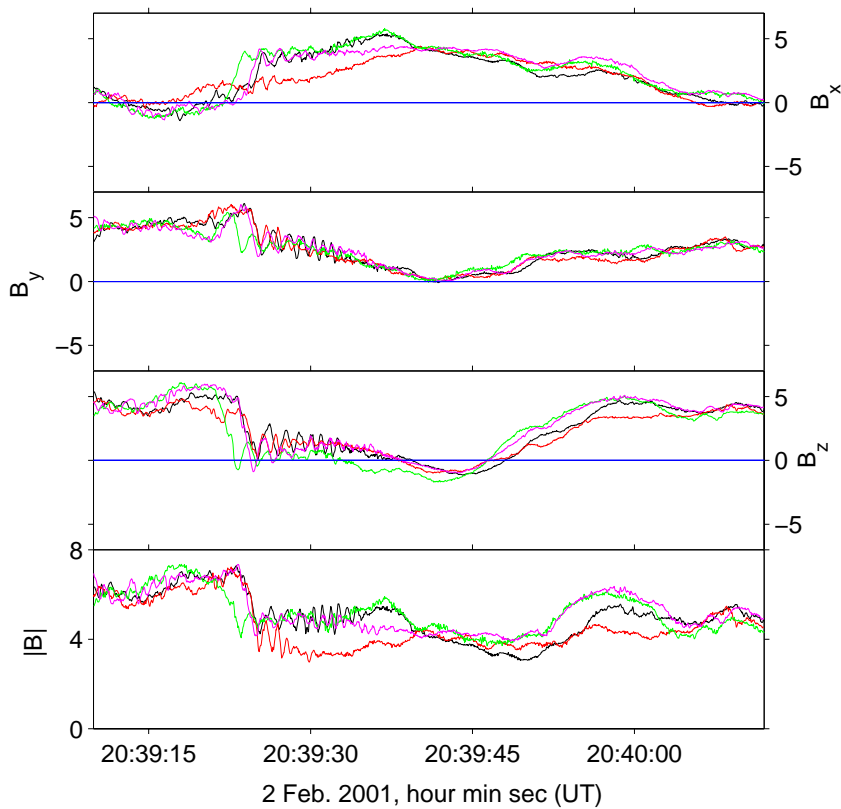

Fig. 11. Magnetic field data at 22 vectors/s in GSE coordinates, in the same format at Fig. 5. The data show a shocklet with an upstream whistler wave.

\subsection{Upstream shocklet characteristics}

Figure 11 shows an example of a steepened upstream ULF wave, called a shocklet, at $\sim 20: 39: 17$ UT. In this case Cluster 3 observes the shocklet first, when it has a slightly shallower gradient and a less well developed high frequency wave than later, when it is encountered by the other three spacecraft, who all observe a steeper leading edge (righthand side) and a better developed high frequency wave train. The delay between the observations at Cluster 3 and at the other three spacecraft is only about $1 \mathrm{~s}$. Since the signature is simultaneous at Cluster 1,2 and 4, this suggests that the normal to the structure lies nearly perpendicular to the plane containing the three spacecraft. Applying timing discontinuity analysis to the leading edge gives a normal of 0.75 , $0.66-0.02$, which is within $10^{\circ}$ of the normal to this plane. The timing analysis normal is within $30^{\circ}$ of the model bow shock normal, a slightly greater angle than observed for either of the SLAMS events. Such an observation is consistent with the simulation of Dubouloz and Scholer (1995), which showed that as ULF waves approached the shock, and developed into SLAMS-like structures, they become more closely aligned with the shock.

The appearance of the shocklet at Cluster 1, 2 and 4 is very similar, although there are small changes in the frequency of the upstream whistler wave. Therefore, although the difference in the signatures at Cluster 3 and the other spacecraft might be spatial in nature, it seems more likely to reflect a temporal evolution on short time scales. The two consecutive structures in Fig. 11 (the shocklet at $\sim 20: 39: 17$ UT and the ULF wave just before 20:39:40 UT) show different space- 
craft ordering across them, indicating that the adjacent structures have different orientations. Minimum variance analysis of the shocklet signature measured by Cluster 2 shows that the ratio between the intermediate and minimum eigenratios is less than 2 , indicating that the minimum variance direction is ill defined in this case. The minimum variance directions calculated for the signature observed by the other three spacecraft differ by $15-20^{\circ}$ but differ from the timing derived estimate for the normal by up to $30^{\circ}$. The discrepancy might arise because the shocklet has only a small variation in magnetic field direction, and hence, the contribution of higher frequency changes increases. This is consistent with the ratio of intermediate to minimum eigenvalues exceeding 2 , but the observed absence of a clear rotation on a hodogram.

Comparison of the timing derived normal with the background magnetic field direction, estimated from ACE data with an appropriate delay, shows that these directions differ by $53^{\circ}$. The delay is calculated using the correspondence between a clear discontinuity observed at Cluster at $\sim 20: 45$ UT, having been observed at ACE 4167s earlier. Using an estimate of the background magnetic field from Cluster during a minute of quiet data immediately following the wave activity, gives a separation of the normal from the background magnetic field of approximately $65^{\circ}$. The three minimum variance directions lie closer to the background magnetic field estimated from the ACE observations, falling in the range of $30-40^{\circ}$. Each estimate of the propagation direction, however, for this example, lies closer to the model bow shock normal than to the background magnetic field vector.

Figure 12 shows a second example of a shocklet, observed about two hours later at $\sim 22: 44$ UT, which appears to be stable during the time in which the tetrahedron traverses the structure. This structure is found within ULF wave activity, just before a very sharp transition into undisturbed solar wind a few minutes later, which is discussed in the next section. Each of the Cluster spacecraft observe a well developed high frequency wave just upstream of the leading edge, although the frequencies of the waves differ slightly between spacecraft. The high frequency waves are again left-hand polarised, consistent with a whistler wave being convected antisunward by the solar wind flow. This example also demonstrates that the orientations of the leading and trailing edges are different. This can be seen from the spacecraft ordering through the signature. The leading edge (right-hand side) is observed first by Cluster 2, and then almost simultaneously by Cluster 1, 3 and 4 . The trailing edge (left-hand side) was observed first by Cluster 2, but the ordering of the other three spacecraft is different. Using discontinuity timing analysis gives leading and trailing edge normal directions which are $\sim 21^{\circ}$ apart. The polarisation of the shocklet signature observed by each spacecraft, excluding the high frequency waves, is right-handed, and there is good agreement between the minimum variance direction and the normal calculated for the leading edge (within $\sim 5$ and $10^{\circ}$ ). That this shocklet shows greater consistency between the minimum variance directions found for each spacecraft and the timing derived normal might arise from the larger amplitude magnetic field ro-

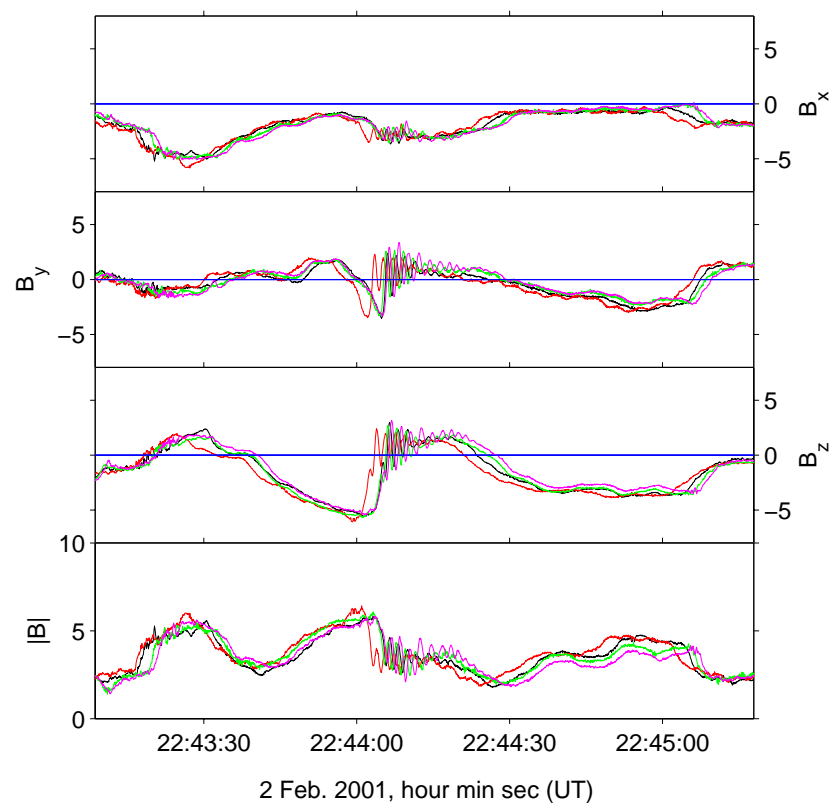

Fig. 12. Magnetic field data at 22 vectors/s in GSE coordinates, in the same format at Fig. 5. The data show a shocklet at 22:44 UT, with an upstream whistler wave.

tation across the wave. The leading edge and minimum variance derived normals lay within $20-30^{\circ}$ of the background magnetic field, estimated using ACE data with an appropriate delay, which is marginally more closely aligned with the background magnetic field than that found for either the previous shocklet examples or either of the SLAMS. Although the location of the satellites far upstream of the expected bow shock position means that the model bow shock normal direction is less relevant, the angle between the leading edge normal and the model bow shock normal was calculated for comparison with the analysis applied to the SLAMS and previous shocklet. The different estimates of the previous shocklet propagation direction lay within $\sim 35^{\circ}$ of the model bow shock normal. A greater angle between the propagation direction estimates and the model shock normal is consistent with the waves further upstream being less influenced by the presence of the shock.

\subsection{Upstream ULF wave characteristics}

In the previous three subsections the characteristics of a small number of magnetic field signatures have been described in detail. For these cases the four satellites observed similar signatures, allowing an estimate to the made of their orientation and motion. Comparison with minimum variance analysis, interpreting the minimum variance direction as the propagation direction that has been used in the past for single spacecraft observations, shows significant differences between the methods. These might arise from the deviations of the structures from planarity on the tetrahedron scales, or from inherent uncertainties in the minimum variance technique, especially for events with small angular changes (Eastwood et al., 


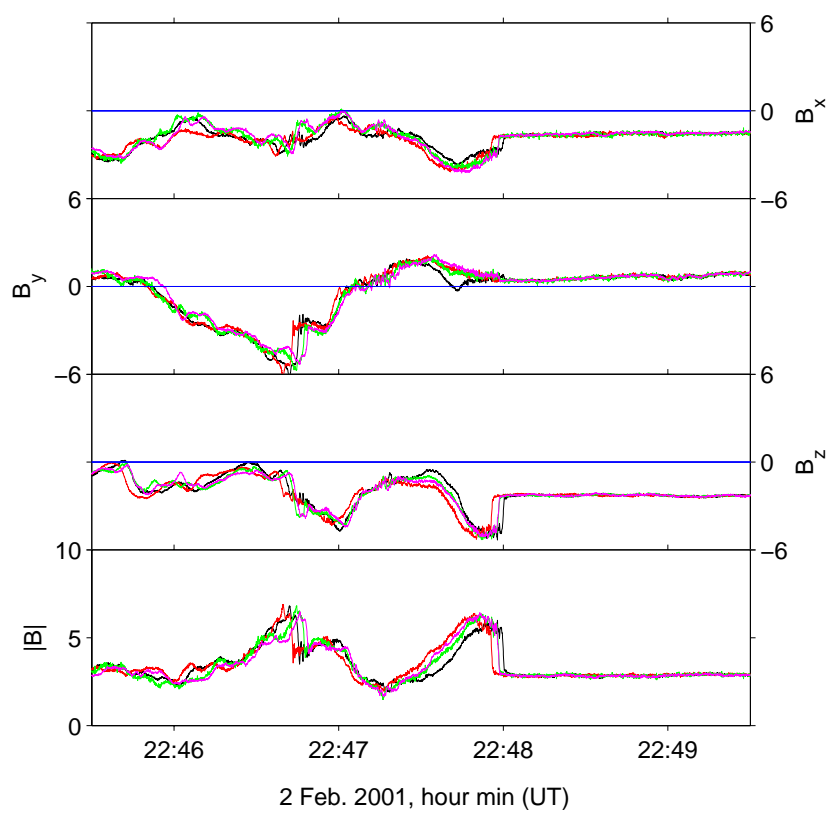

Fig. 13. Magnetic field data at 22 vectors/s in GSE coordinates, in the same format at Fig. 5. The data show an abrupt transition from a region populated by ULF waves and shocklets, to undisturbed solar wind.

2002).

The correlation between the different spacecraft is typically greater during ULF wave activity than is observed for the SLAMS-like signatures, suggesting that the ULF wave scale is larger than that of the magnetic field enhancements, and significantly larger than the spacecraft tetrahedron. This is consistent with the simulations of Dubouloz and Scholer (1995), which showed that differential slowing of the plasma ahead of the shock fragmented the structures that were observed to grow out of the ULF wave field, leading to a smaller scale size. Successive ULF waves, however, typically show different spacecraft ordering, indicating that they do not have the same orientation. Frequently, as can be seen in Figs. 8, 11 and 12, a single ULF wave will also show different spacecraft ordering at the leading and trailing edges, suggesting that the leading and trailing edges can also have different orientations. The smooth, but irregular profile of the ULF waves makes spacecraft timing estimates of their orientation difficult to measure. In addition, minimum variance analysis of the ULF wave field in this case gives minimum variance directions that show a large scatter, both between the different spacecraft observations of any particular wave, and also between successive ULF waves. The scatter varies between waves, but can be large, of the order of $45-90^{\circ}$. This scatter makes it difficult to compare the characteristics of ULF waves and SLAMS-like structures. The ULF waves observed at this shock, however, do not show regular angular variations, and the comparison of the two types of structure would be better compared using an interval when the ULF waves are regular with larger amplitude angular variations, with welldefined and consistent minimum variance directions and a form where timing analysis can be applied.

\subsection{Transition into undisturbed solar wind}

Figure 13 shows a transition from the region containing upstream ULF waves and shocklets, into undisturbed solar wind. It is remarkable for the abrupt nature of the transition, which lasts only for $\sim 1.5 \mathrm{~s}$. The timing analysis normal is found to differ significantly (by $\sim 60^{\circ}$ ), mainly in the $Y-Z_{\mathrm{GSE}}$ direction, from the model bow shock normal, suggesting that, in this case, the orientation of the boundary is not controlled by the bow shock orientation. This signature might be caused by a solar wind discontinuity propagating past the tetrahedron, and we note that it appears to be extremely abrupt. Later transitions are not found to be as sharp and perhaps reflect the spacecraft moving from magnetic field lines which are connected with the shock, to those which are not.

\section{Discussion and conclusions}

We have described some of the features observed at a quasiparallel shock observed by Cluster on 2 February 2001. We described the structure of magnetic field enhancements that have signatures consistent with that expected for SLAMS, and steepened upstream ULF waves (shocklets). We also discussed briefly the characteristics of the ULF waves.

Although the spacecraft observe broadly similar structures, significant variations between the signatures at the different spacecraft are also found. A distinct difference is found between the scale of the upstream ULF waves and shocklets, and the magnetic field enhancements and pulsation regions within the shock transition. The upstream ULF waves appear to be of significantly greater scale, apparently much greater than the tetrahedron scale of $400-1000 \mathrm{~km}$, which is consistent with the expected scales from previous work of $\sim 1 R_{E}$. The scale of the SLAMS-like magnetic field enhancements appears to be shorter than the $0.5-1 R_{E}$ scales found for the upstream ULF wave field (Schwartz, 1991) since significant differences occur between spacecraft. This is consistent with the evolution of SLAMS from the ULF wave field observed in the simulations described by Dubouloz and Scholer (1995), and their subsequent fragmentation by wave front refraction close to the shock. Both shocklets and magnetic field enhancements, however, show varying orientations between successive events, and frequently between the leading and trailing edges of the same event. These results suggest that the structures are not typically planar on the current spacecraft separation scales of a few hundred to a thousand kilometres, which is smaller than the SLAMS scale size of 15-30 ion inertial lengths found by Dubouloz and Scholer (1995).

It is possible that the spacecraft differences observed through the SLAMS arises from the spacecraft sampling significant curvature on the scale of the tetrahedron, and that the orientations of the SLAMS might be ordered on a global 
scale. This is consistent with the picture of a quasi-parallel shock as a patchwork of three-dimensional structures first suggested by Schwartz and Burgess (1991). We note, however, that although the shocklets and ULF waves appear to be of larger scale than the SLAMS, they still appear to have a variety of orientations. If SLAMS do develop from ULF waves, then a variety of orientations might be retained. We also examined the data for signatures consistent with the growth of SLAMS-like structures. Although we have not found clear evidence of such time evolution, it is possible that it is hidden by the spatial variations between the satellites.

In a number of SLAMS-like magnetic field enhancements we find that the angular variation of the magnetic field is better correlated between spacecraft than the magnetic field magnitude, and we find that the $\theta_{B n}$ value, calculated from the local magnetic field and a model bow shock normal, rises to quasi-perpendicular values within magnetic field enhancements at all spacecraft, as expected from previous observations (Mann et al., 1994), despite differences in the profile of $|\mathbf{B}|$. Nevertheless, any combination of the spacecraft data which assumes that the structure is planar, for example, discontinuity timing analysis, must still be applied with caution, since curvature of the structure will bias the results. In addition, the discontinuity timing method is sensitive to small differences in the relative times, making it difficult to apply with confidence unless the four spacecraft observe near identical signatures.

The results of the timing and minimum variance analysis we have presented here suggest that two magnetic field enhancements, both of which have magnetic signatures consistent with SLAMS, have different orientations relative to the model bow shock normal. Clearly a greater number of magnetic field enhancements must be studied before general conclusions can be drawn about SLAMS properties, but we propose that the larger deviation between the estimated propagation direction and the model bow shock normal of the first example might arise from it being embedded in decelerated and deflected solar wind, while the other, which is closely aligned with the model bow shock normal, lies in relatively undisturbed plasma.

Analysis of two examples of upstream shocklets showed that the one located further upstream was less well aligned with the model bow shock normal, consistent with the plasma being less influenced by the shock in this region. Comparison between the orientations of shocklets and SLAMS with respect to the background magnetic field was hampered by a poor estimate of the background field in the region containing the SLAMS-like features, but the results suggested that shocklets might be more closely aligned with the background magnetic field than the SLAMS, consistent with the simulation results of Dubouloz and Scholer (1995). The ULF waves observed on this day showed different orientations at their leading and trailing edges, but had a smooth profile with only small angular variations, and showed variations between spacecraft, making these ULF waves poor candidates for discontinuity timing analysis.

We have demonstrated the potential of Cluster observa- tions for probing the three-dimensional properties of shock structures. A statistical study of the scales and orientations of SLAMS, shocklets and ULF waves with respect to the shock and the magnetic field requires the analysis of multiple events, under conditions when good estimates of these quantities can be made. In addition, multiple observations are required, at a range of tetrahedron scales, in order to examine any dependence of the scale size on direction, such as systematic differences in the scale size along the two directions perpendicular to the nominal shock normal. The future separation strategy for Cluster will lead to quasi-parallel shock observations being made with a tetrahedron of spacecraft separated by only $\sim 100 \mathrm{~km}$. Better correlation should be observed between the different spacecraft under these conditions, allowing for the propagation directions and orientations of the enhancements to be measured more accurately. Later in the mission, larger tetrahedron scales are planned $\left(\sim R_{E}\right.$ scales), which will allow for a better estimation of the scale size of the magnetic field enhancements to be made. We might expect a rapid fall off in the inter-spacecraft correlation if the enhancements are only a little larger than the current tetrahedron, but if the structures are large, with significant spatial evolution across them, a more gradual fall in the correlation as the distance is increased is likely.

The upstream solar wind velocity observed by ACE provides a useful context for this shock, but local plasma measurements from the CIS instrument will be more relevant, allowing us to estimate the plasma frame propagation velocity and to relate the spacecraft separation vectors to the plasma flow direction. A more definitive identification of SLAMS would also be possible. One key question which remains to be answered is the effect which SLAMS have on the plasma, and future collaborative work is planned in order to address this topic.

Acknowledgements. Analysis of Cluster data at Imperial College is supported by PPARC. T. Horbury is supported by a PPARC Fellowship. We gratefully acknowledge the NASA/GSFC CDAWeb for access to the ACE MFI solar wind magnetic field data (provided by N. Ness, Bartol Research Institute) and SWE electron density data (provided by D. J. MacComas, Los Alamos National Laboratory).

Topical Editor G. Chanteur thanks M. Goldstein, A. Mageney and another Referee for their help in evaluating this paper.

\section{References}

Balogh, A., Carr, C. M., Acuña, M. H., Dunlop, M. W., Beek, T. J., Brown, P., Fornacon, K.-H., Georgescu, E., Glassmeier, K.-H., Harris, J., Musmann, G., Oddy, T., and Schwingenschuh, K.: The Cluster Magnetic Field Investigation: overview of in-flight performance and initial results, Ann. Geophysicae, 19, 1207-1217, 2001.

Burgess, D.: Cyclic behaviour at quasi-parallel collisionless shocks, Geophys. Res. Lett., 16, 345-348, 1989.

Dubouloz, N. and Scholer, M.: Two-dimensional simulations of magnetic pulsations upstream of the Earth's bow shock, J. Geophys. Res., 100, 9461-9474, 1995. 
Eastwood, J. P., Balogh, A., Dunlop, M. W., Horbury, T. S., and Dandouras, I.: Cluster observations of fast magnetosonic waves in the terrestrial foreshock, submitted to Geophys. Res. Lett., 2002.

Giacalone, J., Schwartz, S. J., and Burgess, D.: Observations of suprathermal ions in association with SLAMS, Geophys. Res. Lett., 20, 149-152, 1993.

Giacalone, J., Schwartz, S. J., and Burgess, D.: Artificial spacecraft in hybrid simulations of the quasi-parallel Earth's bow shock: analysis of time series versus spatial profiles and a separation strategy for Cluster, Ann. Geophysicae, 12, 591-601, 1994.

Gosling, J. T., Thomsen, M. F., Bame, S. J., and Russell, C. T.: Ion reflection and downstream thermalization at the quasi-parallel bow shock, J. Geophys. Res., 94, 10 027-10 037, 1989.

Greenstadt, E. W., Hoppe, M. M., and Russell, C. T.: Largeamplitude magnetic variations in quasi-parallel shocks: correlation lengths measured by ISEE 1 and 2, Geophys. Res. Lett., 9, 781-784, 1982.

Horbury, T. S., Cargill, P. J., Lucek, E. A., Balogh, A., Dunlop, M. W., Oddy, T. M., Carr, C., Brown, P., Szabo A., and Fornacon, K.-H.: Cluster magnetic field observations of the bowshock: orientation, motion and structure, Ann. Geophys., 19, 1399-1409, 2001.

Horbury, T. S., Cargill, P. J., Lucek, E. A., Eastwood, J., Balogh, A., Dunlop, M. W., Fornacon, K.-H., and Georgescu, E.: Four spacecraft measurements of the quasi-perpendicular terrestrial bowshock: orientation and motion, J. Geophys. Res., doi.: 10.1029/201JA000273, 107, 2002.

Le, G. and Russell, C. T.: A study of the coherence length of ULF waves in the Earth's foreshock, J. Geophys. Res., 95, $10703-$ $10706,1990$.

Le, G. and Russell, C. T.: A study of ULF wave foreshock morphology - I: ULF foreshock boundary, Planet. Space Sci., 40, 1203-1213, 1992a.

Le, G. and Russell, C. T.: A study of ULF wave foreshock morphology - II: Spatial variation of ULF waves, Planet. Space Sci., 40, 1215-1225, 1992b.

Mann, G., Lühr, H., and Baumjohann, W.: Statistical analysis of short large-amplitude magnetic field structures in the vicinity of the quasi-parallel bow shock, J. Geophys. Res., 99, 13315$13323,1994$.
Omidi, N. and Winske, D.: Steepening of kinetic magnetosonic waves into shocklets: simulations and consequences for planetary shocks and comets, J. Geophys. Res., 95, 2281-2300, 1990.

Peredo, M., Slavin, J. A., Mazur, E., and Curtis, S. A.: Threedimensional position and shape of the bow shock and their variation with Alfvénic, sonic and magnetosonic Mach numbers and interplanetary field orientation, J. Geophys. Res., 100, 79087916, 1995.

Scholer, M.: Upstream waves, shocklets, short large-amplitude magnetic structures and the cyclic behaviour of oblique quasiparallel collisionless shocks, J. Geophys. Res., 98, 47-57, 1993.

Scholer, M. and Burgess, D.: The role of upstream waves in supercritical quasi-parallel collisionless shock reformation, J. Geophys. Res., 97, 8319-8326, 1992.

Schwartz, S. J.: An active current sheet in the solar wind, Nature, 318, 269-271, 1985

Schwartz, S. J.: Magnetic field structures and related phenomena at quasi-parallel shocks, Adv. Space Res., 11, 9231-9240, 1991.

Schwartz, S. J.: Shock and discontinuity normals, Mach number, and related parameters, in: Analysis methods for multispacecraft data, (Eds) Paschmann, G. and Daly, P. W., ESA Publications Division, Noordwijk, The Netherlands, 1998.

Schwartz, S. J. and Burgess, D.: Quasi-parallel shocks: a patchwork of three-dimensional structures, Geophys. Res. Lett, 18, 373376, 1991.

Schwartz, S. J., Burgess, D., Wilkinson, W. P., Kessel, R. L., Dunlop, M., and Lühr, H.: Observations of short large-amplitude magnetic structures at a quasi-parallel shock, J. Geophys. Res., 97, 4209-4227, 1992.

Scudder, J. D., Mangeney, A., Lacombe, C., Harvey, C. C., Aggson, T. L., Anderson, R. R., Gosling, J. T., Paschmann, G., and Russell, C. T.: The resolved layer of a collisionless, high $\beta$, supercritical, quasi-perpendicular shock wave 1. Rankine-Hugoniot geometry, currents, and stationarity, J. Geophys. Res., 91, $11019-$ $11052,1986$.

Thomas, V. A., Winske, D., and Omidi, N.: Re-forming supercritical quasi-parallel shocks 1 . One- and two-dimensional simulations, J. Geophys. Res., 95, 18 809-18 819, 1990.

Thomsen, M. F., Gosling, J. T., Bame, S. J., and Russell, C. T.: Magnetic pulsations at the quasi-parallel shock, J. Geophys. Res., 95, 957-966, 1990. 